\title{
The Thickness Dependence of Edge Effect in Thin Insulating Films
}

\author{
Jeong-Myen Song*, Byung-Moo Moon, and Yung-Kwon Sung \\ Department of Electrical Engineering, College of Engineering, Korea University, Anam-dong \\ 5-ga, Seongbuk-gu, Seoul 136-701, Korea \\ *E-mail : jimisong@netian.com
}

(Received 30 May 2003, Accepted 31 July 2003)

\begin{abstract}
This paper deals with the edge effect in thin insulating films, focusing on their dependence on film thickness. The finding is that the electric field is lowered at the edge as the film thickness is reduced, which, in turn, is closely related to dielectric breakdown voltage. In order to analyze this phenomenon, a simple capacitor model is introduced with which dependence of dielectric breakdown voltage around the electrode edge on the film thickness is explained. Due to analytical difficulty to get the expression of electrical field strength at the edge, an equivalent circuit approach is used to find the voltage expression first and then the electric field expression using it. The relation gets to an agreement with the experimental findings shown in the paper. This outcome may be extended to solve similar problems in multi-layer insulating films.
\end{abstract}

Keywords: Thickness dependence, Edge effect, Thin insulating film, Dielectric breakdown

\section{INTRODUCTION}

As semiconductor industry pursues the sub micron technology in fast pace, more strict requirements are being put on high-density integration, functional stability and speed enhancement [1]. In order to meet such requirements, both the process and the materials should be reviewed.

From the process point of view, microelectrode formation technique and multi-layer wiring technology are being emerged as next-generation processes that can go beyond the physical limit of conventional process technology. Materials issues are laid particularly on electrical excellence. One of the obstacles to the sub micron and multi-layer wiring process is thin insulating film thickness with which the reliability and stability must be guaranteed. For example, dielectric strength of the capacitor in ULSI circuit must be good enough to satisfy the design rule of sub micron devices. In particular, the capacitor consists of thin dielectric layer between the metal layers of which edges are the points where maximum electric field takes place. As we know, high electric field at the edge may cause the breakdown of thin insulating layer or corona discharge, which may result in the root cause of the device malfunction or life degradation due to deterioration of the film quality. Nowadays dielectric strength of the layer becomes the key item to be developed urgently especially in high-density integration devices process $[2,3]$.

In this paper, $\mathrm{Al}$ electrodes are positioned on both sides of $\mathrm{SiO}_{2}$ layer so that parallel capacitor model is selected to find the analytical expression of electric field at arbitrary points inside $\mathrm{SiO}_{2}$ layer. The concept of equivalent circuit model is employed, being distributed in the space along the extended line from the electrode edge because it is almost impossible to analytically get the electric field expression at the electrode edge. This formula generates the electric fields on the different edges with various film thicknesses and also the equations between electric field and insulating film thickness $[4,5]$. Comparison with experimental data will be given for the validation of the work.

\section{ELECTRIC FIELD EXPRESSION}

\subsection{Derivation electric field formula inside the capacitor}

First of all, simplify the structure of capacitor by placing metal electrodes on the top and bottom of insulating film with thickness $c$, and set the shape of electrodes as the rectangle with width a and height $b$. In addition, apply the voltage and set the electric potential in such a way that the upper electrode has higher electric 
potential than the lower electrode, and set the surface charge density of the upper electrode as $\sigma$ and that of the lower electrode as $-\sigma$, dielectric constant of $\mathrm{SiO}_{2}$ as $\varepsilon$. Then, the electric field at some $\operatorname{point}(\mathrm{x}, \mathrm{y}, \mathrm{z})$ can be obtained by integrating micro electric field of micro electric charge $\sigma$ dx'dy' over each electrode. We obtain electric field from the lower electrode and from the upper electrode, by applying the superposition principle of electric field vector, and obtain the total combination electrode field formula by summing them up.

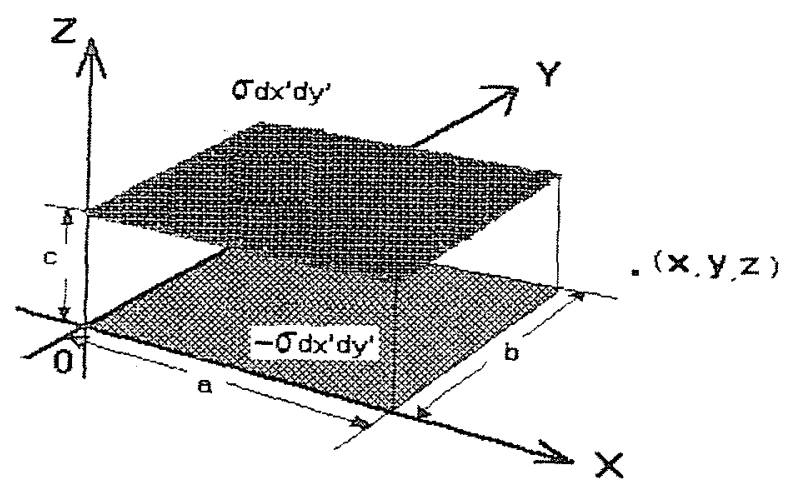

Fig. 1. Schematic model of the capacitor with insulating film embedded between metal plates.

Basic formula used for deriving electric field is the same as the following known formula.

$$
\begin{aligned}
& d \hat{E}(x, y, z)=\frac{\sigma d x^{\prime} d y^{\prime}}{4 \pi \varepsilon} \frac{\left(x-x^{\prime}\right) \bar{a} x+\left(y-y^{\prime}\right) \hat{a} y+\left(z-z^{\prime}\right) \bar{a} z}{\left(\left(x-x^{\prime}\right)^{2}+\left(y-y^{\prime}\right)^{2}+\left(z-z^{\prime}\right)^{2}\right)^{3 / 2}} \\
& \hat{E}(x, y, z)=\int d \hat{E}(x, y, z)
\end{aligned}
$$

Each component of the electric field and the strength of total electric field are as shown below.

(The proportion constants such as $\sigma$ and $4 \pi \varepsilon$ were omitted)

$$
\begin{aligned}
E_{x}(x, y, z) & =\ln \left|\frac{(y-b)+\sqrt{x^{2}+(y-b)^{2}+(z-c)^{2}}}{(y-b)+\sqrt{(x-a)^{2}+(y-b)^{2}+(z-c)^{2}}}\right| \\
& -\ln \left|\frac{y+\sqrt{x^{2}+y^{2}+(z-c)^{2}}}{y+\sqrt{(x-a)^{2}+y^{2}+(z-c)^{2}}}\right| \\
& -\ln \left|\frac{(y-b)+\sqrt{x^{2}+(y-b)^{2}+z^{2}}}{(y-b)+\sqrt{(x-a)^{2}+(y-b)^{2}+z^{2}}}\right| \\
& +\ln \left|\frac{y+\sqrt{x^{2}+y^{2}+z^{2}}}{y+\sqrt{(x-a)^{2}+y^{2}+z^{2}}}\right|
\end{aligned}
$$

$$
\begin{aligned}
& E_{y}(x, y, z)= \ln \left|\frac{(x-a)+\sqrt{(x-a)^{2}+y^{2}+(z-c)^{2}}}{(x-a)+\sqrt{(x-a)^{2}+(y-b)^{2}+(z-c)^{2}}}\right| \\
&-\ln \left|\frac{(x-a)+\sqrt{(x-a)^{2}+y^{2}}+z^{2}}{(x-a)+\sqrt{(x-a)^{2}+(y-b)^{2}}+z^{2}}\right| \\
& E_{z}(x, y, z)= \arctan \frac{x y}{(z-c) \sqrt{x^{2}+y^{2}+(z-c)^{2}}} \\
&+\arctan \frac{(x-a)(y-b)}{(z-c) \sqrt{(x-a)^{2}+(y-b)^{2}+(z-c)^{2}}} \\
&-\arctan \frac{x(y-b)}{(z-c) \sqrt{x^{2}+(y-b)^{2}+(z-c)^{2}}} \\
&-\arctan \frac{(x-a) y}{(z-c) \sqrt{(x-a)^{2}+y^{2}+(z-c)^{2}}} \\
&-\arctan \frac{x y}{z \sqrt{x^{2}+y^{2}+z^{2}}} \\
&-\arctan \frac{(x-a)(y-b)}{z \sqrt{(x-a)^{2}+(y-b)^{2}+z^{2}}} \\
&+\arctan \frac{x(y-b)}{z \sqrt{x^{2}+(y-b)^{2}+z^{2}}} \\
&+\arctan \frac{(x-a) y}{z \sqrt{(x-a)^{2}+y^{2}+z^{2}}} \\
& E(x, y, z)=\sqrt{E_{x}^{2}+E_{y}^{2}+E_{z}^{2}}
\end{aligned}
$$

Electric field strength at the different height inside the capacitor is shown in Fig. 2. The parameters are chosen with $a=10, b=5, c=1$ to have the thin film condition. The result show, that the electric field is uniform inside the capacitor because the electric field strength is the same at any location inside the capacitor.

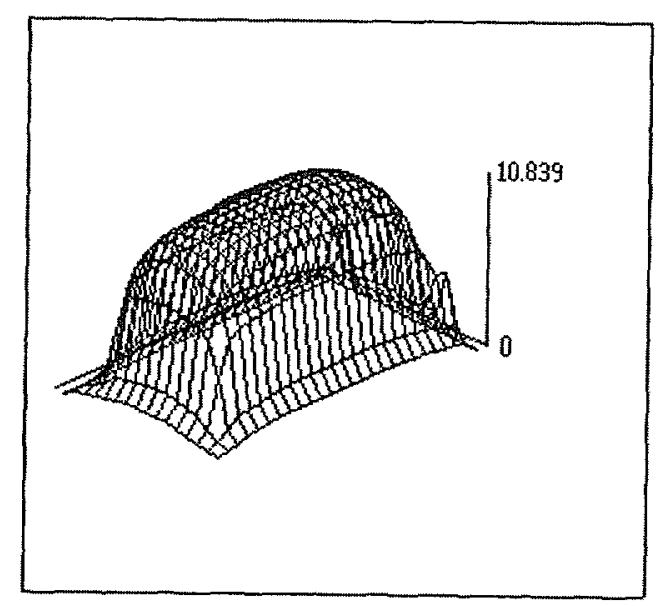

(a) In case of height close to anode $(z=0.9 c)$ 


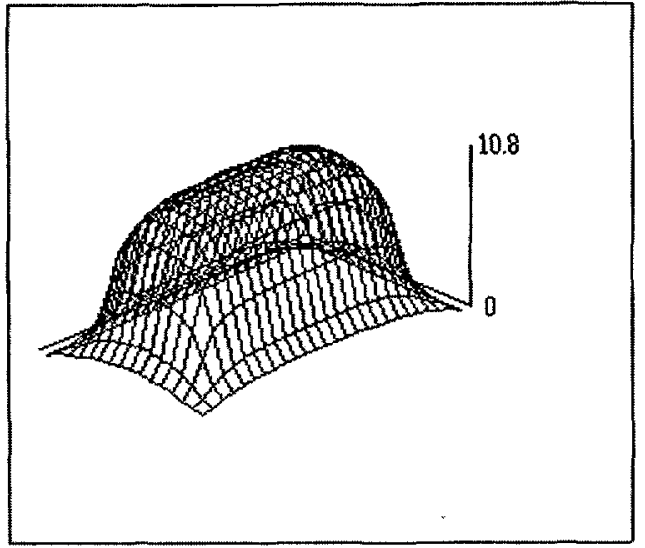

(b) In case of mid-point between poles $(z=0.5 c)$

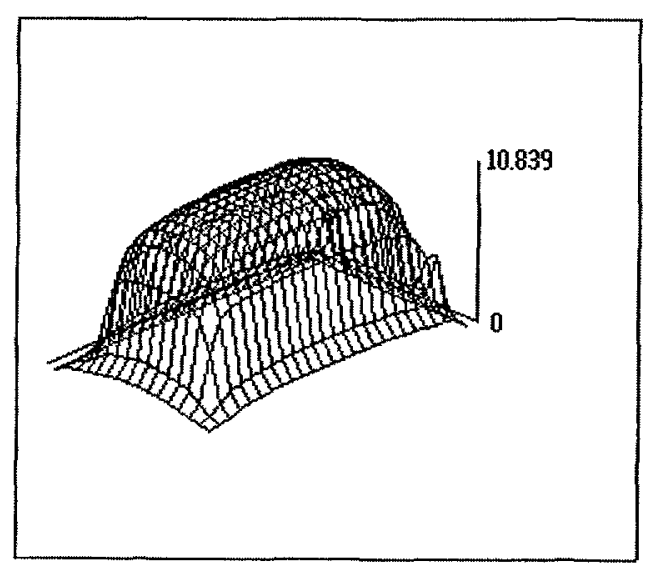

(c) In case of height close to cathode $(z=0.1 c)$

Fig. 2. Electric field strength outside and inside the capacitor.

\subsection{Derivation of electric field formula at the edge}

Since electric field strength cannot be analytically deduced at the edge of electrode, a simple model is set by introducing the concept of equivalent circuit constant integer, which is distributed throughout the space that lies on the extended line from the electrode edge. Voltage and current relations of the transmission line are applied to obtain the strength of electric field near the edge of the electrode.

Assuming the voltage given to the electrode as $V_{m}$ and the voltage on the electrode surface as constant, the voltage at edge, that is, one end of the electrode can be regarded as $V_{m}$. Now, expressing the space on the horizontally extended line from the electrode edge in terms of equivalent circuit, it can be expressed as shown in Fig. 3.

Assuming that space on the extended line from the edge is long enough, and if the voltage at the point, distance $\ell$ away from the edge, is $V(\ell)$, the voltage $V(\ell)$ satisfies the following formula.

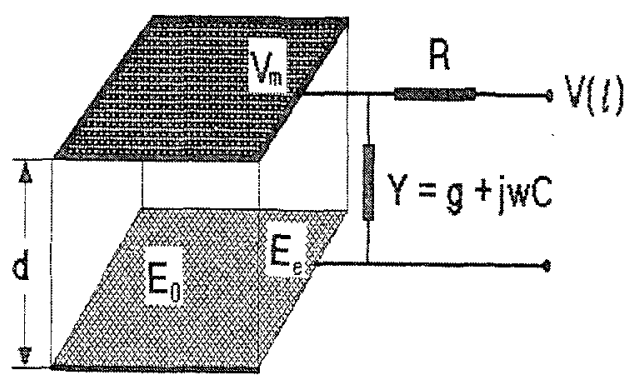

Fig. 3. Equivalent circuit on horizontally extended line from the electrode edge.

$$
\begin{aligned}
& \frac{d V}{d l}=-R I \\
& \frac{d I}{d l}=-Y I
\end{aligned}
$$

We obtain the voltage formula only by differentiating the above 2 formula with respect to $\ell$,

$$
\frac{d^{2} V}{d l^{2}}=R Y V
$$

The solution of this differential equation can be found as

$$
V(\ell)=A \exp (a \ell)+B \exp (-a \ell)
$$

To decide coefficient $\mathrm{A}$ and $\mathrm{B}$ the boundary condition, $V(\ell)=V_{m}, V(\infty)=0$, is applied. Then voltage formula can be expressed as Eq. 7 since $\mathrm{A}=0$ and $\mathrm{B}=\mathrm{V}$.

$$
V(l)=V_{m} \text { exp }(-a l)
$$

From this equation

$$
a=\sqrt{R Y}=\sqrt{R(g+j w C}=\alpha+j \beta
$$

Then,

$$
V(l)=V_{m} \exp (-a l)=V_{m} \exp (-\alpha l) \exp (-j \beta l)
$$

Since $\exp (-j \beta l)$ of Eq. 9 determines the phase, we just consider the magnitude of $V(l)$.

$$
V(l)=V_{m} \exp (-\alpha l)
$$

Above equation can be obtained, where, 


$$
a=\sqrt{\frac{1}{2}\left(R \sqrt{g^{2}+(w C)^{2}}+g\right)}
$$

From Eq. 8, this value is

$$
\begin{aligned}
a & =\alpha+j \beta=\sqrt{R(g+j w C)} \\
& =\sqrt{R\left(\sqrt{g^{2}+(w C)^{2}(\cos \theta+j \sin \theta)}\right.} \\
& =\sqrt{R}\left(g^{2}+(w C)^{2}\right)^{\frac{1}{4}} \sqrt{e^{j \theta}} \\
& =\sqrt{R}\left(g^{2}+(w C)^{2}\right)^{\frac{1}{4}} e^{j \frac{\theta}{2}} \\
& =\sqrt{R}\left(g^{2}+(w C)^{2}\right)^{\frac{1}{4}}\left(\cos \frac{\theta}{2}+j \sin \frac{\theta}{2}\right) .
\end{aligned}
$$

Using the equation, $\tan \theta=\frac{w C}{g}$

$$
a=\sqrt{R}\left(g^{2}+(w C)^{2}\right)^{\frac{1}{4}} \cos \frac{\theta}{2} .
$$

From $\tan \theta=\frac{w C}{g}$ in Eq. 13

$$
\cos \theta=\frac{g}{\sqrt{g^{2}+(w C)^{2}}}
$$

And using cosine formula, we obtain

$$
\begin{aligned}
& \cos ^{2} \frac{\theta}{2}=\frac{1+\cos \theta}{2}=\frac{1+\frac{g}{\sqrt{g^{2}+(w C)^{2}}}}{2} \\
& =\frac{\frac{1}{2} g+\frac{1}{2} \sqrt{g^{2}+(w C)^{2}}}{\sqrt{g^{2}+(w C)^{2}}} .
\end{aligned}
$$

Therefore,

$$
\cos \frac{\theta}{2}=\frac{\left(\frac{1}{2} g+\frac{1}{2} \sqrt{g^{2}+(w C)^{2}}\right)^{\frac{1}{2}}}{\left(g^{2}+(w C)^{2}\right)^{\frac{1}{2}}}
$$

Inserting Eq. 15 to Eq. 14,

$$
\begin{aligned}
& a=\sqrt{R}\left(g^{2}+(w C)^{2}\right)^{\frac{1}{4}} \frac{\left(\frac{1}{2} g+\frac{1}{2} \sqrt{g^{2}+(w C)^{2}}\right)^{\frac{1}{2}}}{\left(g^{2}+(w C)^{2}\right)^{\frac{1}{4}}} \\
& =\left(\frac{1}{2} R \sqrt{g^{2}+(w C)^{2}}+\frac{1}{2} R g\right)^{\frac{1}{2}} .
\end{aligned}
$$

This is Eq. 11. If the layer thickness $d$ is constant, and setting voltage at $\ell=L$ assumed to be the edge, as $V_{e}$, the voltage is $V_{m} \exp (-\alpha L)$ and the strength of electric field at the edge, $\mathrm{E}$ is

$$
E=\frac{V_{m} \exp (-\alpha L)}{d}
$$

Can be obtained.

\subsection{Derivation of relation between film thickness} and electric field strength at edge.

Electric field strength at the edge given in Eq. 16 is the case where the film thickness is constant. Dependence on the film thickness can be deduced through the following procedure. $\alpha$ value in Eq.16 is determined by $\mathrm{g}$ and $\mathrm{C}$, and since the capacitor area is constant and dielectric constant of insulating film is same, $g$ and $C$ depend on film thickness only;

$$
\begin{aligned}
& g=\frac{A}{d} \\
& C=\frac{B}{d} \quad \text { (A and B are constant) }
\end{aligned}
$$

Using the above relations and $\alpha$ value expressed in the function of film thickness, and applying the Eq. 17.1 and Eq. 17.2 to Eq. 11 , and assuming all values except $\mathrm{d}$ value as constant $M$, we find

$$
\alpha=\frac{M}{\sqrt{d}}
$$

The expression between film thickness and electric field strength at the edge can be obtained by inserting Eq. 18 to $\mathrm{Eq} .16$ as below.

$$
E=\frac{V_{m}}{d} \exp \left(-\frac{K}{\sqrt{d}}\right) \quad(\mathrm{K}=\mathrm{ML})
$$

Figure 4 shows the electric field strength over the film thickness for each $\mathrm{K}$ value.

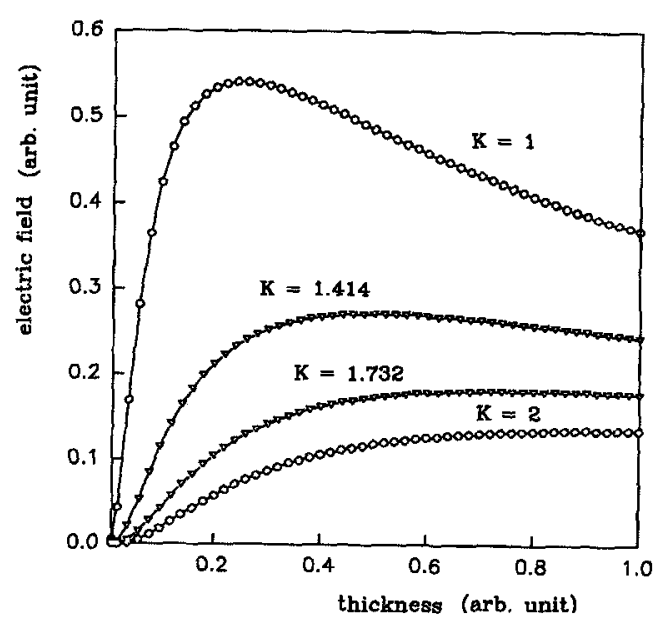

Fig. 4. Electric field strength at the edge depending on film thickness. 
From this result, it was found that the electric field strength is decreased as the film thickness becomes thinner. The $\mathrm{K}$ value involves physical conditions of the insulating film being determined by the capacitance representing the charge storage capacity and by the conductance representing the leakage current. This value therefore, varies with the film types and film processes. Fig. 5 and Fig. 6 show the dependence of breakdown strength of insulating film on film thickness. The breakdown strength increases as the film becomes thinner [6,7]. This reflects the fact that dielectric breakdown may occur with higher voltage since the strength of electric field is decreased under the same voltage as the film becomes thinner as shown in fig. 4 . This verifies that our expression is in good agreement with the experimental results.

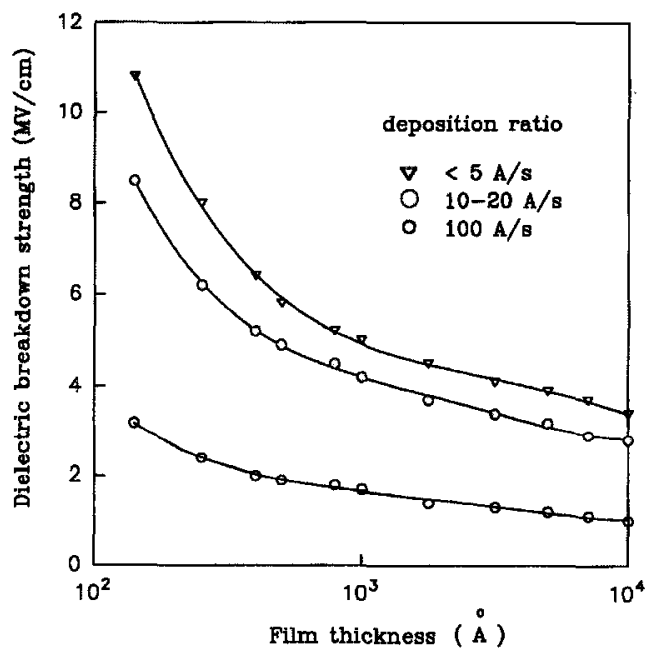

Fig. 5. Insulating breakdown strength over film thickness at $\mathrm{SiO}$ film deposited with different deposition speed [6].

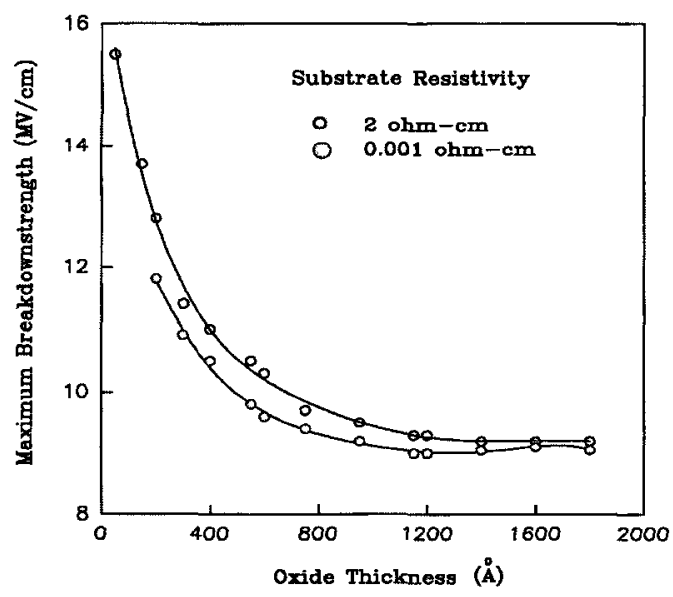

Fig. 6. Insulating breakdown strength over oxidizing film thickness with different resistivity [7].

\section{CONCLUSION}

It is widely known that the dielectric strength of an insulating film decreases inside the capacitor as the insulating film becomes thinner, together with edge effect indicating that the electric field of the capacitor is concentrated on the edge of electrode. However, the change in the electric field strength at edge of electrode over the insulating film thickness has not attracted much attention. In this context, we selected a simple capacitor model for dependence of dielectric breakdown strength around electrode edge on the film thickness, which is not properly highlighted, and obtained electric field formula through a simple derivation process. The simple equation shows the change of electric field over the film thickness through the voltage formula at the edge by introducing the concept of equivalent circuit of transmission line.

As a result, our work finds that the electric field at the edge decreases as film becomes thinner. It was also verified that this relation under constant voltage matches with the experimental result that higher voltage should be given to generate dielectric breakdown. This work should be reviewed and compared more carefully with actual measurement data related to multiple wiring and multi-layer insulating film structure.

\section{REFERENCES}

[1] C. W. Ho, D. A. Chance, C. H. Bajorek, and R. E. Acosta, "The thin-film module as a highperformance semiconductor package", IBM J. Res. Develop., Vol. 26, No. 3, p. 286, 1982.

[2] C. M. Osburn and D. W. Ormond, "Dielectric breakdown in silicon dioxide films on silicon (1)", J. Electrochem.Soc., Vol. 119, No. 5, p. 591, 1972.

[3] C. M. Osburn and N. J. Chou, "Accelerated dielectric breakdown of silicon dioxide films", J. Electrochem. Soc., Vol. 120, No. 10, p. 1377, 1973.

[4] Munir H. Nayfeh and Morton K. Brussel, "Electricity and magnetism (in Korean)", 1990.

[5] S. B. Park, "Theory of alternating current circuit", Mun Un Dang, p. 86, 1983.

[6] Y. Inagaki, N. Tsuchida, and S. Nitta, "Dielectric breakdown properties of evaporated $\mathrm{SiO}$ films", J. I. E. E. J, Vol. 89-9, No. 972, p. 206, 1969.

[7] C. M. Osburn and D. W. Ormond, "Dielectric breakdown in silicon dioxide films on silicon (2)", J. Electrochem. Soc., Vol. 119, No. 5, p. 597, 1972. 